\title{
A multi-angle plane wave imaging approach for high frequency 2D flow visualization in small animals: simulation study in the murine arterial system
}

\author{
Abigail Swillens, Darya Shcherbakova \\ Bram Trachet, Patrick Segers \\ IBiTech-bioMMeda \\ Ghent University, Ghent, Belgium \\ Email: abigail.swillens@ugent.be
}

\author{
Ingvild Kinn Ekroll \\ Lasse Lovstakken \\ Department of Circulation and Medical Imaging \\ NTNU, Trondheim, Norway
}

\begin{abstract}
To preclinically investigate the role of hemodynamics in atherogenesis, mouse models are particularly useful due to the rapid disease development. As such, murine blood flow visualization has become an important tool, with current US systems equipped with traditional 1D flow imaging techniques, lacking spatial and/or temporal resolution to accurately resolve in-vivo flow fields. Hence, we investigated multi-angle plane wave imaging for ultrafast, $2 \mathrm{D}$ vector flow visualization and compared this approach with conventional pulsed Doppler in the setting of a mouse aorta with abdominal aortic aneurysm. For this purpose, we used a multiphysics model which allowed direct comparison of synthetic US images with the true flow field behind the image. In case of the abdominal aorta, we showed the mean flow estimation improved 9\% when using 2D vector Doppler compared to conventional Doppler, but still underestimated the true flow because the full spatial velocity distribution remained unknown. We also evaluated a more challenging measurement location, the mesenteric artery (aortic side branch), often assessed in a short-axis view close to the origin of the branch to avoid the smaller dimensions downstream. Even so, complex out-ofplane flow dynamics hampered a reliable flow assessment for both techniques. Hence, both cases illustrated the need for 3D vascular imaging, allowing acquisition of the full 3D spatial velocity profile.
\end{abstract}

\section{INTRODUCTION}

While atherosclerosis is the main cause of cardiovascular diseases, its aetiology is not fully understood yet. However, it is believed disturbed blood flow dynamics, e.g. low and oscillatory wall shear stress, might play an important role in the origin and progression of the disease. To preclinically investigate the hemodynamical processes involved in atherogenesis, mouse models of cardiovascular pathologies can be particularly useful due to the rapid disease development. As such, murine blood flow visualization has become an important tool, which recently became available due to the development of highfrequency ultrasound (US) probes [1].

However, current small animal systems are equipped with traditional 1D flow imaging techniques such as pulsed wave Doppler (PWD) and color flow imaging, suffering from insufficient spatial and/or temporal resolution to accurately resolve in-vivo flow fields. Furthermore, the extrapolation of these imaging methods from the human to the murine setting is not always straightforward as some physiological parameters inherently differ between both species. For instance, the 10 times faster heart rate in mice only allows to obtain 3-4 color flow images per cardiac cycle in case of a conventional imaging setup, i.e. linear scanning with focused transmit beams. Such low frame rates do not allow to portray the quick spatial and temporal variations in the murine arterial flow field.

These limitations have paved the way for computational fluid dynamics (CFD), a numerical technique allowing assessment of the desired hemodynamical information (flow velocities and pressures) and its derived parameters (e.g. wall shear stress, vorticity, ...) in arbitrarily complex geometries at high accuracy and at any desired resolution. However, CFD is also subject to 2 important limitations since it outcome depends on (i) the accuracy of the 3D reconstructed flow domain, typically based on CT/MRI-scans, (ii) a-priori knowledge on the hemodynamic conditions (flow and/or pressure) at the boundaries of the simulated flow domain, often assessed via ultrasonic flow measurements.

Hence, our aim is to investigate a recently proposed ([2], [3], [4]) multi-angle, plane wave imaging setup for 2D vector flow visualization at a high frame rate (ultrafast, i.e. order of several hundreds of $\mathrm{Hz}$ ), serving a 2-fold purpose:

1) Improving the accuracy of CFD by providing better boundary conditions

2) Assessing its feasibility for improved direct, in-vivo hemodynamical imaging.

In particular, we will compare the performance of volume flow estimation derived from this ultrafast 2D vector Doppler approach versus conventional pulsed wave Doppler in the setting of a mouse aorta. For this purpose, we will use a multiphysics model integrating computational fluid dynamics with US simulations (Field II [5], [6]), by propagating the point scatterers representing the blood flow in Field II according to complex blood flow fields obtained from CFD simulations. This has the advantage that synthetic US images can be created, where the true flow field behind the image is fully known [7].

Hence, in this work, we will first perform CFD simulations in a murine aorta representative of a genetically modified mouse with induced abdominal aortic aneurysm (AAA), serving as an input to Field II. Next, we will compare the performance of conventional Doppler and ultrafast 2D flow imaging for volume flow assessment through direct comparison 


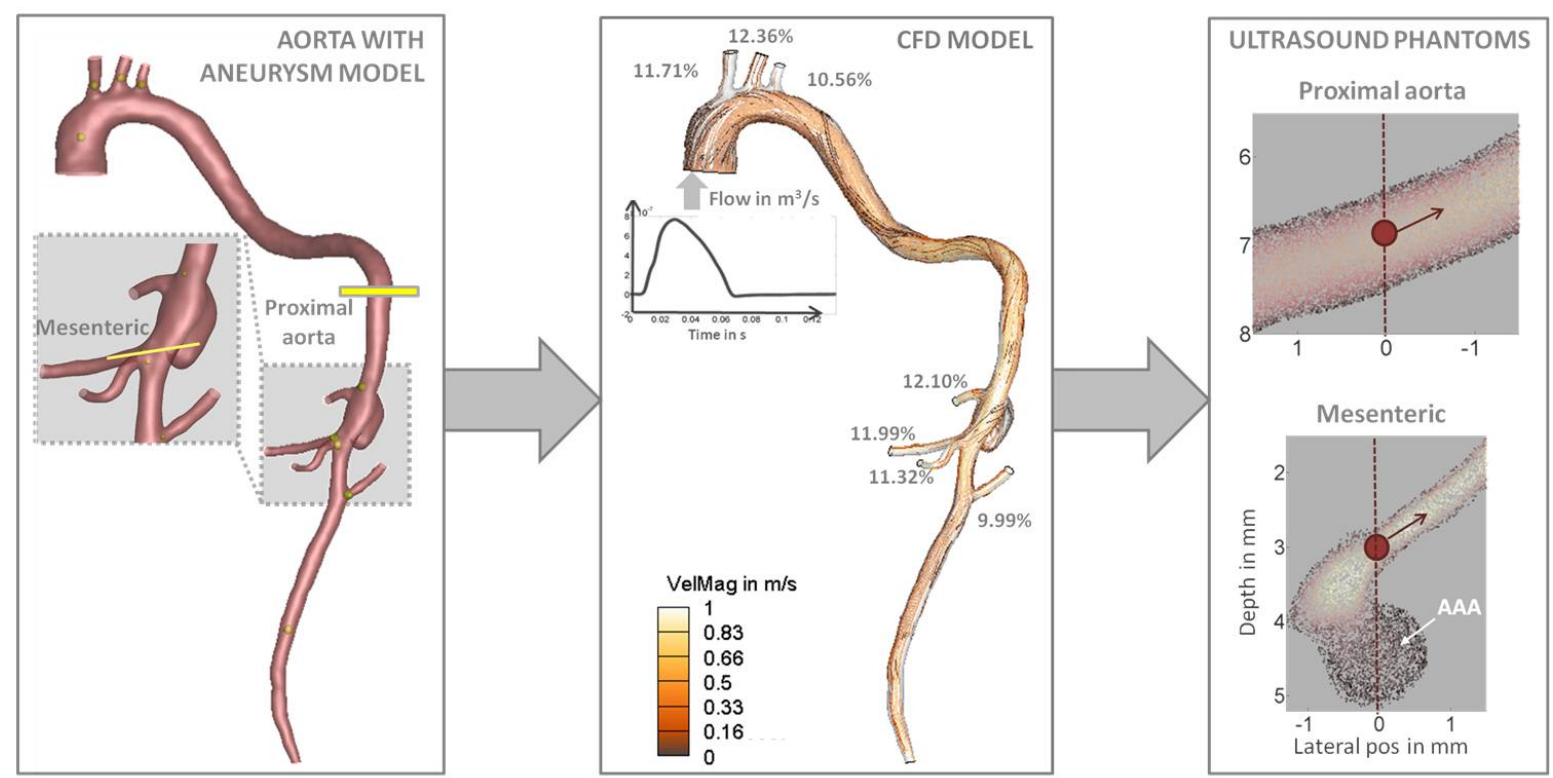

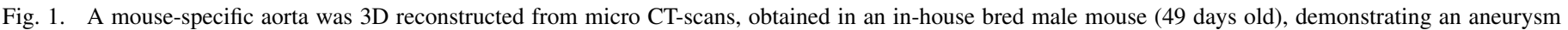
in the abdominal and ascending aorta. The CFD flow patterns were used to move point scatterers in Field II.

of the simulated US flow data with the true blood flow from the CFD-simulations.

\section{METHODS}

\section{A. Multiphysics simulations in the murine aorta}

1) 3D-reconstruction murine aorta: A mouse-specific aorta was $3 \mathrm{D}$ reconstructed from a set of micro CT-scans (left panel of fig.1), obtained in an in-house bred male mouse (49 days old), demonstrating an abdominal aortic aneurysm (AAA), induced after 28 days of angiotensin II uptake via an implanted osmotic pump [8]. Based on the reconstructed images, the aorta was semi-automatically segmented to select the contrastenhanced lumen using Mimics (Materialise, Leuven, Belgium). A tetrahedral volume mesh was created in Gambit (ANSYS, Canonsburg, PA, USA).

2) CFD-simulation: The flow fields in this mouse-specific aortic model were obtained using the CFD software Fluent (ANSYS, Canonsburg, PA, USA). At the inlet of this model, a flat velocity profile was imposed with a maximum temporal velocity of $0.2 \mathrm{~m} / \mathrm{s}$ (middle panel fig.1). We chose Murray's law to determine the outflow ratio's between the 8 different outlet branches (middle panel fig.1):

$$
\frac{Q_{m}}{Q_{s}}=\frac{r_{m}^{2}}{r_{s}^{2}}
$$

with $Q_{m}$ and $r_{m}$ the volumetric flow and radius of the mother branch, and $Q_{s}$ and $r_{s}$ the flow and radius of the side branch. For each branch, this equation was solved for $Q_{s}$, starting from the aortic inlet towards the distal abdominal aorta, the latter receiving the remaining flow fraction.

3) Ultrasound simulation: To allow flexible modeling of the ultrasonic imaging process, we used Field II ([5], [6]), which represents the imaged blood flow as point scatterers, on which the US waves reflect. To obtain a proper random distribution inside a complex arterial network, we developed an algorithm based on the open-source software VTK, creating points in a box surrounding the complete geometry and removing those outside the lumen based on geometrical criteria. The remaining scatterers' positions were updated for each simulated beam according to the CFD flow fields [7]. We modeled a high-frequency linear array probe representative of the MS 550D transducer of the Vevo 2100 system (Visualsonics, Toronto, Canada), emitting sinusoidal pulses at $32 \mathrm{MHz}$.

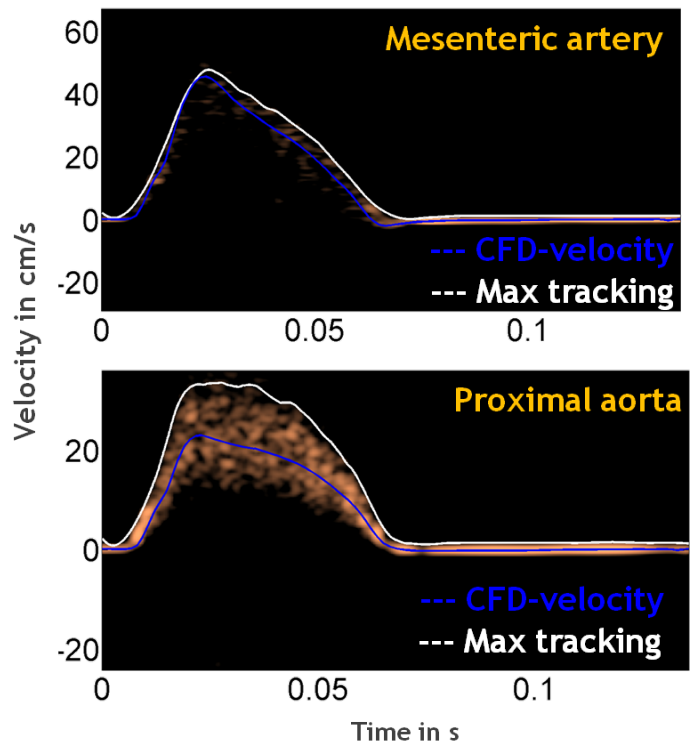

Fig. 2. Synthetic pulsed wave Doppler spectra in proximal AA and mesenterica: (i) blue $=$ CFD ground truth, (ii) white $=$ max tracing of spectrum. 

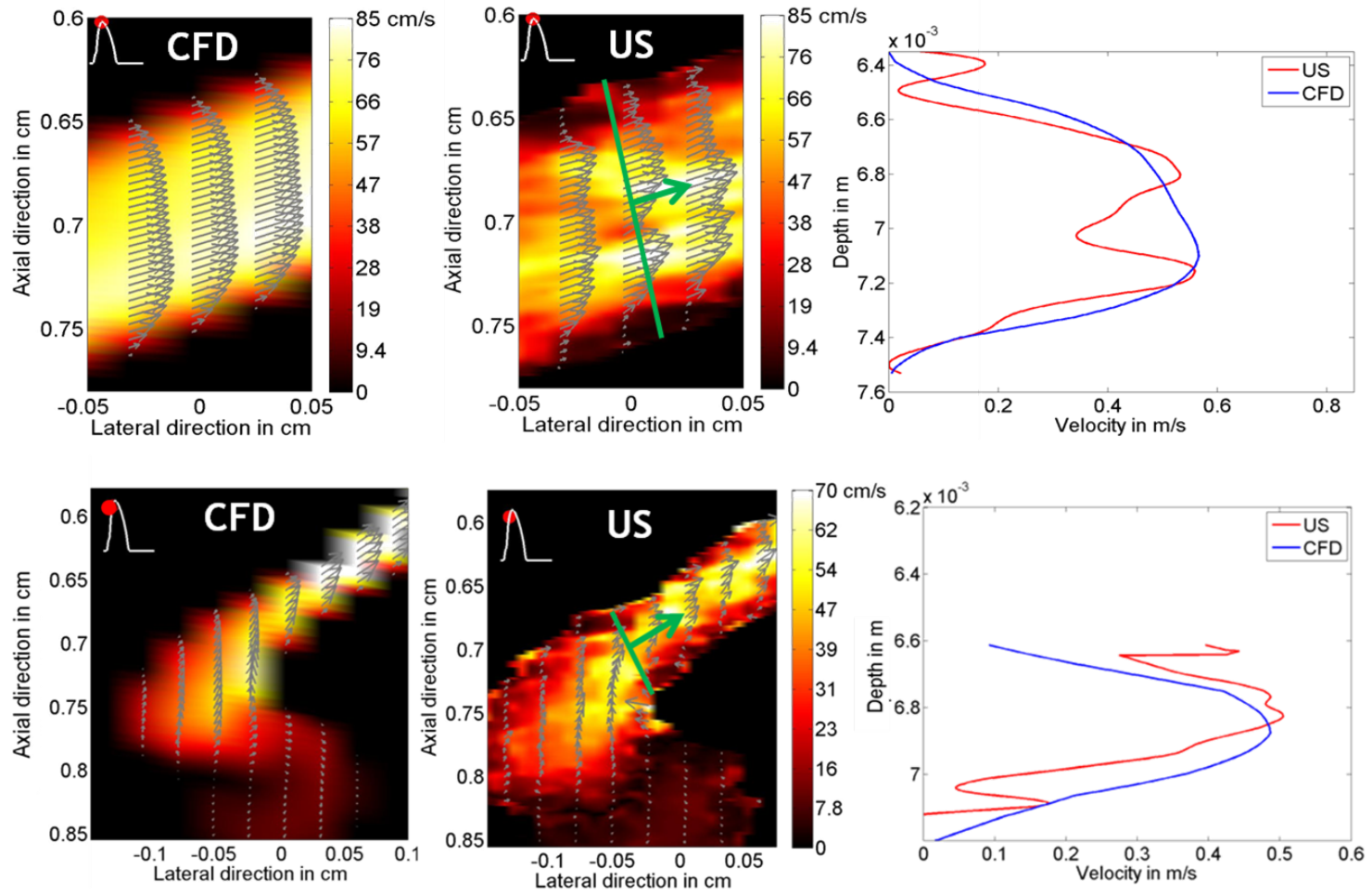

Fig. 3. 2D velocity images in the proximal AA (upper) and mesenterica (below) for the CFD ground truth (left) and synthetic ultrasound (middle) at peak systole. In the right panels, the velocity profile is shown along the green line which is used for subsequent volume flow estimation.

TABLE I. IMAGING SETUP PULSED WAVE DOPPLER

\begin{tabular}{|c|c|}
\hline Pulsed wave Doppler & Proximal AA /mesenteric \\
\hline Focus on $\mathrm{Tx}$ & $6.8 / 3 \mathrm{~mm}$ \\
\hline$F_{\#}$ on $\mathrm{Tx}$ & 2.5 \\
\hline$F_{\#}$ on $\mathrm{Rx}$ & 1.5 \\
\hline
\end{tabular}

\section{B. Ultrasonic volume flow estimation}

We compared the outcome of volume flow estimation derived from: (i) conventional PWD and (ii) ultrafast $2 D$ vector Doppler. We focused on 2 scanning locations: (i) a long-axis view of the proximal abdominal aorta (AA) and (ii) a short-axis view of the mesenterica. The corresponding scatterer phantoms are visualized in fig.1 (right panel), together with the location of the sample volume (red dot) and US-beam (dashed line) for the PWD acquisition. For both scanning schemes, the pulse repetition frequency (PRF) was set to 25 and $40 \mathrm{kHz}$ for the proximal AA and mesenterica respectively.

For the PWD assessment, the sonograms were obtained in the sample volume of interest via Fourier analysis. The mean frequency of the power density spectra was calculated and converted to velocity $\left(v_{P W D}\right)$ via the classical Doppler equation. For the $2 D$ vector Doppler approach, $2 \mathrm{D}$ velocity information was obtained through angled plane wave emission in an interleaved scheme with opposite steering angles (angles of $\pm 10^{\circ}$ ). The $2 \mathrm{D}$ velocity vectors were retrieved by combining the autocorrelation estimates from both steering angles in the whole region simultaneously, facilitated by parallel beamforming on receive (using the same steering angles as on transmit) allowing an ultrafast scanning. Further details on the pulsed Doppler and 2D vector Doppler setup are respectively mentioned in table I and II.

TABLE II. IMAGING SETUP PLANE WAVE IMAGING

\begin{tabular}{|c|c|}
\hline Plane wave imaging & Proximal AA /mesenteric \\
\hline Parallel receive beams & 200 \\
\hline Angle & $10^{\circ}$ \\
\hline Packetsize for autocorrelation & 10 \\
\hline Apodization on Tx & yes \\
\hline
\end{tabular}

Based on the acquired 1D and 2D velocity info, the instantaneous volume flow $\mathrm{Q}(\mathrm{t})$ through the cross-section of interest A was calculated, theoretically defined as the integration of the spatial velocity profile over the area:

$$
Q(t)=\int_{A}(\vec{v}(t) \cdot \vec{n}) d A=\int_{A} v_{n}(t) d A
$$

with $\vec{v}(t)$ the 3D-velocity vector in a certain point of the cross-section, $\vec{n}$ the normal of the cross-section A and $v_{n}$ the projection of the velocity vector onto the normal. Evaluation of this surface integral was performed via 2 different strategies:

(i) For the PWD assessment, it was assumed the sample volume was positioned at the location of the spatial maximum of the velocity profile. Volume flow was retrieved after angle correction and the assumption of a spatial velocity distribution:

$$
Q(t)=\beta * v_{n, \max }(t) * A
$$

with $\beta$ 0.5, equivalent to parabolic flow. The angle-corrected velocity $v_{n}(t)$ was obtained by correcting the Doppler velocity 


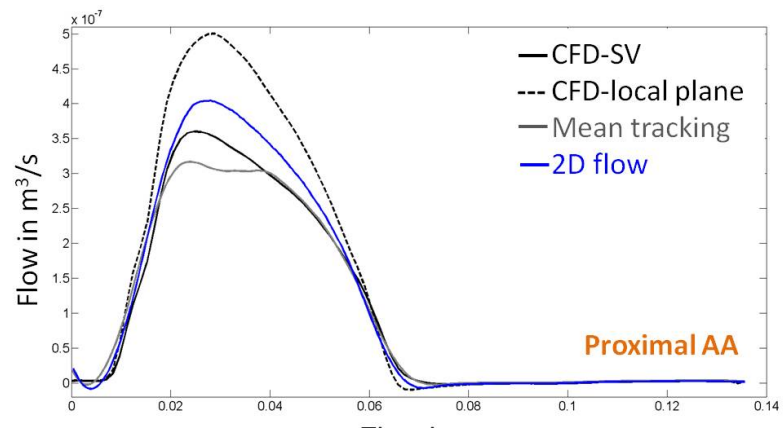

Time in s

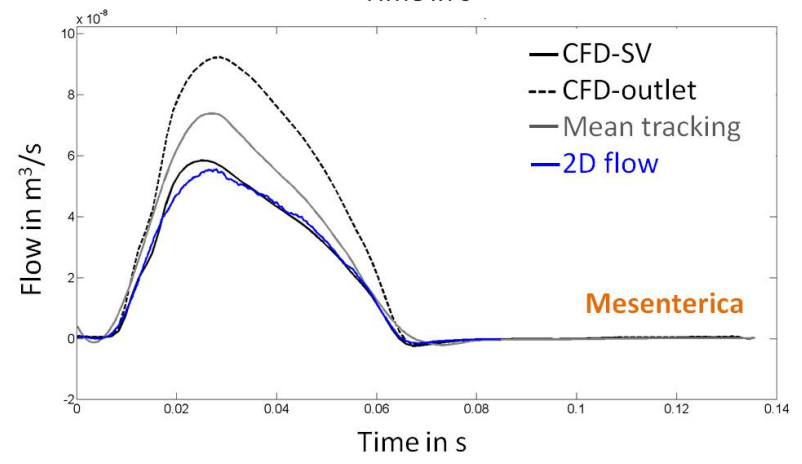

Fig. 4. Comparison in volume flow estimation obtained via PWD and 2D vector Doppler assessment.

$v_{P W D}(t)$ as: $v_{n}(t)=\frac{v_{P W D}(t)}{\cos (\theta)}$, with $\theta$ the angle between the US beam and the assumed flow direction, set to $70^{\circ}$ and $59^{\circ}$ in the proximal AA and mesenterica respectively.

(ii) For the $2 D$ vector Doppler approach, velocity information was acquired along a line in the imaging plane (indicated in green in fig.3), at each time point (542 and 339 frames for the proximal AA and mesenterica respectively). These 2D velocity data were projected on the area's normal and the mean of this velocity distribution was used to compute flow as $Q=v_{\text {mean }} * A$

\section{RESUlTS \& DiscusSiON}

The synthetic Doppler spectra for the proximal AA and mesenterica are shown in fig.2. The blue curve represents the ground truth, i.e. the CFD velocity at the location of the sample volume projected along the US beam direction. The white curve corresponds to the maximum frequency tracking of the spectrum. The spectrum in the mesenteric artery is deteriorated which could be attributed to high velocities and the large outof-plane flow component.

Via 2D vector Doppler, we visualized the 2D flow field in the proximal AA and mesenterica at peak systole (fig.3). The correspondence between the synthetic US and CFD data can be readily appreciated, though a spatially varying deviation is present in the velocity magnitude as well as deviations in the flow direction in case of the mesenterica. The velocity profile along the green line, used to compute the volume flow, is shown on the right (red) and compared to the ground truth (blue), confirming the effect of the inherent statistics of the velocity estimation.

The volume flow for the proximal AA and mesenterica is displayed in fig.4. For the proximal $A A$, it is clear that the PWD method (solid gray curve, mean tracking) seriously underestimates the true flow in the cross-section (black dashed curve, CFD-local plane). However, it matches reasonably well with the CFD flow obtained after applying the flow measurement procedure for PWD data on the CFD-velocity in the corresponding sample volume (solid black curve, $C F D$ $S V$ ). When using 2D velocity info (blue curve), the deviation on the mean flow measurement improves from $24 \%$ to $15 \%$ (compared to CFD-local plane). The remaining underestimation can be largely attributed to a skewed velocity profile in the aorta. For the mesenterica, the mean tracking of the Doppler spectrum (gray curve) results in a slight overestimation of the $C F D-S V$ flow (solid black curve), which is better captured by the 2D flow measurement (blue). Both the PWD and vector Doppler procedure seriously underestimate the flow further downstream of the branch (dashed black curve, CFD-outlet) due to the complex hemodynamics in this measurement view.

\section{CONCLUSION}

A multiphysics modeling approach was applied to investigate volume flow estimation in the murine arterial system, obtained with the PWD and ultrafast 2D vector Doppler approach, though without the effects of clutter filtering. In case of quite laminar flow patterns (proximal AA), the study showed a markedly improvement when using 2D vector Doppler data, though still underestimating because the full spatial velocity distribution remained unknown. Assessing flow in the aortic side branches is challenging due to the small dimensions and hence, a short-axis view is typically applied, with the sample volume close to the origin of the branch. Even so, the complex out-of-plane flow dynamics hampered correct assessment. Both cases illustrated the need for 3D vascular imaging modalities, allowing acquisition of the full 3D spatial velocity profile.

\section{ACKNOWLEDGMENT}

We thank Andrew Needles for his input on the VEVO 2100 system. A. Swillens and D. Shcherbakova are grant recipients of the Research Foundation Flanders (FWO).

\section{REFERENCES}

[1] D.Christopher, "A high-frequency pulsed-wave doppler ultrasound system for the detection and imaging of blood flow in the microcirculation," $U M B$, vol. 23, no. 7, pp. 997-15, 1997.

[2] M. Tanter, "Ultrafast compound imaging for $2 \mathrm{~d}$ motion vector estimation: Application to transient elastography," IEEE TUFFC, vol. 49, no. 10, pp. 1363-1374, 2002.

[3] J. Udesen, "High frame-rate blood vector velocity imaging using plane waves: Simulations and preliminary experiments," IEEE TUFFC, vol. 55, no. 8, pp. 1729-1743, 2008.

[4] I. K. Ekroll, "Simultaneous quantification of flow and tissue velocities based on multi-angle plane wave imaging," IEEE TUFFC, vol. 60, no. 4, pp. 727-738, 2013.

[5] J. Jensen, "A new calculation procedure for spatial impulse responses in ultrasound," JASA, vol. 105, p. 32663274, 1999.

[6] J. Jensen, "Field: A program for simulating ultrasound systems," Med \& Biol Eng Comp, vol. 34, pp. 351-352, 1996.

[7] A. Swillens, "Ultrasound simulation of complex flow velocity fields based on computational fluid dynamics," IEEE TUFFC, vol. 56, no. 3, pp. 546-556, 2009.

[8] B.Trachet, "The impact of simplified boundary conditions and aortic arch inclusion on cfd simulations in the mouse aorta: a comparison with mousespecific reference data," J Biom Eng, vol. 133, no. 13, 2011. 